\title{
Comparison of self-reported and accelerometer- assessed measurements of physical activity according to socio-demographic characteristics in Korean adults
}

\author{
Seung Won Lee ${ }^{1,2}$, Jee-Seon Shim ${ }^{2,3}$, Bo Mi Song ${ }^{1,2}$, Ho Jae Lee ${ }^{2}$, Hye Yoon Bae ${ }^{2}$, Ji Hye Park ${ }^{1,2}$, \\ Hye Rin Choi ${ }^{1,2}$, Jae Won Yang ${ }^{1,2}$, Ji Eun Heo ${ }^{1,2}$, So Mi Jemma Cho, ${ }^{1,2}$, Ga Bin Lee ${ }^{1,2}$, \\ Diana Huanan Hidalgo ${ }^{1,2}$, Tae-Hoon Kim ${ }^{4}$ Kyung Soo Chung ${ }^{5}$, Hyeon Chang Kim ${ }^{2,3}$ \\ ${ }^{1}$ Department of Public Health, Yonsei University of Graduate School, Seoul, Korea; ${ }^{2}$ Cardiovascular and Metabolic Diseases Etiology Research \\ Center, Yonsei University College of Medicine, Seoul, Korea; ${ }^{3}$ Department of Preventive Medicine, Yonsei University College of Medicine, Seoul, \\ Korea; ${ }^{4}$ Department of Cardiology, Severance Cardiovascular Hospital, Yonsei University College of Medicine, Seoul, Korea; ${ }^{5}$ Department of \\ Internal Medicine, Institute of Chest Disease, Yonsei University College of Medicine, Seoul, Korea
}

\begin{abstract}
OBJECTIVES: Previous studies have shown relatively low correlations between self-reported and accelerometer-assessed physical activity (PA). However, this association differs by socio-demographic factors, and this relationship has not been fully investigated in the general population. Thus, we investigated the correlation between self-reported and accelerometer-assessed PA and whether it differed by demographic and socioeconomic factors among the Korean general population.
\end{abstract}

METHODS: This cross-sectional study included 623 participants (203 men and 420 women) aged 30 to 64 years, who completed a PA questionnaire and wore a wrist-worn accelerometer on the non-dominant wrist for 7 days. We examined the agreement for metabolic equivalent task minutes per week (MET-min/wk) between the 2 measures and calculated Spearman correlation coefficients according to demographic and socioeconomic factors.

RESULTS: The kappa coefficient between tertiles of self-reported and accelerometer-assessed total MET-min/wk was 0.16 in the total population, suggesting overall poor agreement. The correlation coefficient between the 2 measurements was 0.26 ( $p<0.001$ ) in the total population, and the correlation tended to decrease with increasing age ( $\mathrm{p}$ for trend $<0.001)$ and depression scores $(\mathrm{p}$ for trend $<0.001)$.

CONCLUSIONS: We found a low correlation between self-reported and accelerometer-assessed PA among healthy Korean adults, and the correlation decreased with age and depression score. When studying PA using accelerometers and/or questionnaires, age and depression need to be considered, as should differences between self-reported and accelerometer-assessed PA.

KEY WORDS: Physical activity, Accelerometer, Questionnaire, Korea

\section{Correspondence: Hyeon Chang Kim}

Department of Preventive Medicine, Yonsei University College of Medicine, 50 Yonsei-ro, Seodaemun-gu, Seoul 03722, Korea

E-mail: hckim@yuhs.ac

Received: Sep 12, 2018 / Accepted: Nov 29, 2018 / Published: Nov 29, 2018

This article is available from: http://e-epih.org/

(C) This is an open-access article distributed under the terms of the Creative Commons Attribution License (http://creativecommons.org/licenses/by/4.0/), which permits unrestricted use, distribution, and reproduction in any medium, provided the original work is properly cited.

(C) 2018, Korean Society of Epidemiology

\section{INTRODUCTION}

Physical activity (PA) is an important modifiable risk factor for cardiovascular disease (CVD), diabetes mellitus, falls, osteoporosis, obesity, some cancers, and mortality [1-4]. Epidemiologic studies usually assess PA by self-reported questionnaires for practical reasons [5]. However, self-reported data are vulnerable to reporting bias [6]. Moreover, light-intensity activities are hard to recall and tend to be under-reported $[7,8]$. These errors in the measurement of PA might attenuate estimates of the effect of PA on health-related outcomes [9]. Objective measures, such as pedome- 
ters and accelerometers, have emerged as an alternative to solve these problems. Accelerometers can provide objective estimates of the duration and intensity of PA [5,10]. Agreement between questionnaire- and accelerometer-assessed PA was remarkably low in previous studies $[5,11]$. Recall and response bias in questionnaire surveys might be largely responsible for discrepancies between these 2 measures [12]. Previous studies reported that these biases can be influenced by demographic factors, socioeconomic status, and health status $[13,14]$. Furthermore, accelerometers attached to the upper body cannot detect certain activities that only use the lower body, such as weightlifting and cycling. Previous studies also reported that the association between questionnaire- and accelerometer-assessed PA differed by age, gender, ethnicity, socioeconomic status, and level of PA $[5,13,14]$. However, there are limited data on the association between questionnaire- and accelerometer-assessed levels of PA in the Korean population. Thus, we compared questionnaire-based and accelerometer-assessed PA among Korean adults, and investigated whether socio-demographic factors affected the correlation between these 2 measurements of PA.

\section{MATERIALS AND METHODS}

\section{Study population}

This study was conducted utilizing baseline data from the Cardiovascular and Metabolic Diseases Etiology Research Center (CMERC) study, which was launched in 2013. The CMERC study consists of 2 prospective cohorts: a general population cohort (the CMERC cohort) and a cohort of high-risk patients (the CMERCHI cohort). The data collection procedures of the CMERC cohort have been described elsewhere in detail [15]. Wrist-worn accelerometry was performed in a subsample of the participants in the CMERC cohort operated by the Department of Preventive Medicine, Yonsei University College of Medicine. They were relatively healthy people without a history of major CVD, such as myocardial infarction or stroke, when they were enrolled in the CMERC cohort.

Between December 2013 and September 2017, a total of 738 individuals participated in PA measurements using a 3-dimensional accelerometer. They all completed health questionnaires and health examinations using an identical protocol. In the current study, participants were included if they had available PA data from the accelerometer for at least $16 \mathrm{hr} / \mathrm{d}$ for 7 days. After excluding 101 persons with invalid accelerometer data and 4 persons with unreliable accelerometer data, 623 participants (203 men and 420 women) aged 30 to 64 years old were included in the current analysis. All participants provided written informed consent, and the institutional review board of Severance Hospital, Yonsei University Health System, Seoul, Korea (4-2013-0661) approved the study protocol.

\section{Measurement of physical activity by questionnaire}

For the questionnaire-based assessment of PA, we used a Kore- an version of the International Physical Activity Questionnaire (IPAQ)-Short Form, which asks for the frequency of each activity and the duration thereof during the past 7 days [16]. The short form records activities at 4 intensity levels: (1) vigorous-intensity activity such as aerobics, (2) moderate-intensity activity such as leisure cycling, (3) walking, and (4) sitting. According to the IPAQ scoring protocol [17], participants' responses were converted to metabolic equivalent task minutes per week (MET-min/wk). Using the Ainsworth et al. [18] compendium, an average MET score was derived for each type of activity [18]. The following values were used for the analysis of IPAQ data: walking = 3.3 METs, moderate $\mathrm{PA}=4.0 \mathrm{MET}$, vigorous $\mathrm{PA}=8.0 \mathrm{MET}$, and total PA MET$\mathrm{min} / \mathrm{wk}=$ sum of walking+moderate +vigorous MET-min/wk scores. A previous study reported Spearman rho coefficients and kappa values of test-retest reliability in Korean adults aged 15-69 years of 0.427-0.646 (median, 0.542) and 0.365-0.620 (median, 0.471), respectively [19]. The kappa values were greater than 0.4 in 5 of the 7 questionnaires. In a study of elderly individuals, the Spearman rho coefficients and kappa values of test-retest reliability for 5 parameters (vigorous days, vigorous minutes, moderate days, moderate minutes, and walk days) were $0.299-0.605$ and 0.307-0.418, respectively [16].

\section{Measurement of physical activity by accelerometer}

For the accelerometer-based assessment of PA, a wrist-worn triaxial accelerometer (GENEActiv; Activinsights Ltd., Kimbolton, UK) was used. The accelerometers were pre-programmed with a $100-\mathrm{Hz}$ sampling frequency and participants were asked to wear the accelerometer on their non-dominant wrist for 7 consecutive days and nights. The raw data were downloaded to a personal computer using the software supplied by the manufacturer (GENEActiv version 2.2) and transformed into 1-minute epoch files. To obtain values including the duration of each activity and MET score for the current analyses, we used the GENEActiv macro file 'General physical activity' version 1.8 , which was previously validated $[20,21]$. All participants continued to wear accelerometers at night.

\section{Other questionnaire data}

The CMERC cohort study collected demographic and socioeconomic data on gender, age, education, marital status, and household income [15]. Marital status was defined as living with a partner or not. Education was categorized as primary school or below, lower secondary school, higher secondary school or university degree or higher. Income level was categorized as lower, middle, or upper based on tertile values of annual household income.

Cognitive function was only assessed in participants aged 50 years or older, using the Korean version of the Mini-Mental State Estimation (MMSE) for dementia screening [22]. MMSE scores range from 0 to 30 , with a higher score indicating better cognitive performance. We used a cutoff of 26 to categorize participants as having cognitive impairment, as in previous studies [13,23]. Depressive symptoms were assessed using the Korean version of the 
Beck Depression Inventory-II (BDI) [24,25].

\section{Anthropometric measurements}

Standing height was measured to the nearest $0.1 \mathrm{~cm}$ using a stadiometer (DS-102, Jenix, Seoul, Korea). Body weight was measured to the nearest $0.1 \mathrm{~kg}$ on a digital scale (DB-150, CAS, Seongnam, Korea) according to a predefined protocol [15]. Body mass index (BMI) was calculated as an individual's body weight in kilograms divided by his or her height in meters squared.

\section{Statistical analyses}

Gender differences were analyzed using the independent $t$-test or the Wilcoxon rank-sum test for continuous variables and the chi-square test for categorical variables. In order to investigate agreement between PA (MET-min/wk) measured by the questionnaire and the accelerometer, we compared tertile values for the 2 measurements using the kappa index. The correlation between questionnaire and accelerometer-assessed PA was evaluated using Spearman correlation coefficients, along with Bland-Altman plots. These analyses were conducted for the total population, and then separately for the following categories: gender, age

Table 1. General characteristics of the study population

\begin{tabular}{|c|c|c|c|c|}
\hline Variables & Total $(n=623)$ & Men $(n=203)$ & Women $(n=420)$ & p-value ${ }^{1}$ \\
\hline Age (yr) & $53.0 \pm 9.1$ & $52.5 \pm 10.2$ & $53.3 \pm 8.6$ & 0.31 \\
\hline BMI $\left(\mathrm{kg} / \mathrm{m}^{2}\right)$ & $23.8 \pm 2.9$ & $24.5 \pm 2.7$ & $23.5 \pm 3.0$ & $<0.001$ \\
\hline \multicolumn{5}{|l|}{ Marital status } \\
\hline Married/cohabiting & $544(87.3)$ & $195(96.1)$ & $349(83.1)$ & \multirow[t]{2}{*}{$<0.001$} \\
\hline Single & $79(12.7)$ & $8(3.9)$ & $71(16.9)$ & \\
\hline \multicolumn{5}{|l|}{ Education } \\
\hline Secondary school or below & $318(51.0)$ & $73(36.0)$ & $245(58.3)$ & \multirow[t]{2}{*}{$<0.001$} \\
\hline University degree or more & $305(49.0)$ & $130(64.0)$ & $175(41.7)$ & \\
\hline \multicolumn{5}{|l|}{ Income } \\
\hline Lower & $195(31.3)$ & $46(22.7)$ & $149(35.5)$ & \multirow[t]{3}{*}{0.005} \\
\hline Middle & $193(31.0)$ & $69(34.0)$ & $124(29.5)$ & \\
\hline Upper & $235(37.7)$ & $88(43.4)$ & $147(35.0)$ & \\
\hline \multicolumn{5}{|l|}{ MMSE score } \\
\hline$<26$ & $225(36.1)$ & $76(37.4)$ & $149(35.5)$ & \multirow[t]{2}{*}{0.63} \\
\hline$\geq 26$ & $398(63.9)$ & $127(62.6)$ & $271(64.5)$ & \\
\hline \multicolumn{5}{|l|}{ BDI score } \\
\hline None (0-13) & $474(76.1)$ & $170(83.7)$ & $304(72.4)$ & \multirow[t]{4}{*}{0.01} \\
\hline Mild (14-19) & $93(14.9)$ & $24(11.8)$ & $69(16.4)$ & \\
\hline Moderate (20-28) & $43(6.9)$ & $7(3.5)$ & $36(8.6)$ & \\
\hline Severe (29-63) & $13(2.1)$ & $2(1.0)$ & $11(2.6)$ & \\
\hline \multicolumn{5}{|c|}{ Physical activity by questionnaire (min/wk) } \\
\hline Sitting time & $2,691 \pm 1,395$ & $3,004 \pm 1,518.6$ & $2,539 \pm 1,306$ & $<0.001$ \\
\hline Walking time & $280[120-600]$ & $270[120-525]$ & 300 [120-600] & 0.55 \\
\hline Moderate activity time & $0[0-140]$ & $0[0-180]$ & $0[0-120]$ & 0.06 \\
\hline Vigorous activity time & $0[0-0]$ & $0[0-120]$ & $0[0-0]$ & $<0.001$ \\
\hline Total MET & $1,590[693-3,228]$ & $1,782[716-3,626]$ & $1,560[693-3,113]$ & 0.08 \\
\hline People with moderate activity $\geq 60$ & $45(7.2)$ & $21(10.3)$ & $24(5.7)$ & 0.05 \\
\hline People with vigorous activity $\geq 600$ & $121(19.4)$ & $60(29.6)$ & $61(14.5)$ & $<0.001$ \\
\hline \multicolumn{5}{|c|}{ Physical activity by accelerometer (min/wk) } \\
\hline Sedentary activity time & $4,605 \pm 791.4$ & $4,860 \pm 822.1$ & $4,482 \pm 747$ & $<0.001$ \\
\hline Light activity time & 780 [601-964] & $637[493-896]$ & 825 [694-999] & $<0.001$ \\
\hline Moderate activity time & $1,129[842-1,495]$ & $996[759-1,355]$ & $1,175[907-1,535]$ & $<0.001$ \\
\hline Vigorous activity time & $28[13-58]$ & $40[20-82]$ & $23[11-50]$ & $<0.001$ \\
\hline Total MET & $12,457[11,053-14,044]$ & $12,211[10,861-13,765]$ & $12,595[11,295-14,114]$ & 0.07 \\
\hline People with moderate activity $\geq 60$ & $570(91.5)$ & $179(88.2)$ & $391(93.1)$ & 0.06 \\
\hline People with vigorous activity $\geq 600$ & $524(84.1)$ & $185(9.1)$ & $339(80.7)$ & $<0.001$ \\
\hline
\end{tabular}

Values are presented as mean \pm standard deviation, median [interquartile range], or number (\%).

BMI, body mass index; MMSE, Mini-Mental State Estimation; BDI, Beck Depression Inventory-II; MET, metabolic equivalent task.

${ }^{1} \mathrm{p}$-values were derived from the independent $\mathrm{t}$-test, the Wilcoxon rank sum test, or chi-square test. 
group (i.e., 30-39, 40-49, 50-59, and $\geq 60$ years), BMI category, marital status, education, household income, cognitive function, and prevalent depression.

Since the Spearman correlation coefficient is equal to the slope of the regression between the ranked values of the 2 measures, gender differences were tested by regressing the gender-specific rank of accelerometer-assessed total MET-min/wk on the genderspecific rank of questionnaire-assessed total MET-min/wk together with the interaction term (gender $\times$ rank of questionnaire-assessed PAs) using a linear model, similarly to a previous study [14].

Table 2. Agreement between tertiles of self-reported and accelerometer-assessed total MET-min/wk

\begin{tabular}{lcccc}
\hline \multirow{2}{*}{$\begin{array}{c}\text { Self-reported } \\
\text { MET-min/wk }\end{array}$} & \multicolumn{2}{c}{ Accelerometer-measured } & MET-min/wk & \multirow{2}{*}{$\begin{array}{c}\text { Kappa } \\
\text { statistic }\end{array}$} \\
\cline { 3 - 4 } $\begin{array}{l}\text { Total }(\mathrm{n}=623) \\
\text { Lower }\end{array}$ & $91(44.4)$ & $61(28.8)$ & $49(23.8)$ & 0.16 \\
Middle & $67(32.7)$ & $80(37.7)$ & $69(33.5)$ & \\
Upper & $47(22.9)$ & $71(33.5)$ & $88(42.7)$ & \\
Men $(\mathrm{n}=203)$ & & & & \\
Lower & $27(40.9)$ & $19(27.1)$ & $18(26.9)$ & 0.16 \\
Middle & $26(39.4)$ & $27(38.6)$ & $19(28.4)$ & \\
Upper & $13(19.7)$ & $24(34.3)$ & $30(44.8)$ & \\
Women $(\mathrm{n}=420)$ & & & & \\
Lower & $63(45.7)$ & $43(30.1)$ & $30(21.6)$ & 0.19 \\
Middle & $45(32.6)$ & $53(37.1)$ & $47(33.8)$ & \\
Upper & $30(21.7)$ & $47(32.9)$ & $62(44.6)$ & \\
\hline
\end{tabular}

MET, metabolic equivalent task.
The p-value for interaction was used to test whether the correlation between questionnaire-based and accelerometer-assessed PA differed by gender. This analysis was repeated for the demographic and socioeconomic variables under consideration. For age, BMI, educational level, income, and BDI score, the p-value for the trend across categories was also calculated by fitting a linear group interaction term with the rank of MET-min/wk. All analyses were performed using SAS version 9.4 (SAS Institute Inc., Cary, NC, USA). All statistical tests were 2 -sided and p-values less than 0.05 were considered to indicate statistical significance.

\section{RESULTS}

The general characteristics of the study population are presented in Table 1 . The mean age was 52.5 years in men and 53.3 years in women. The median (interquartile range) of total MET-min/ wk was $1,590(693-3,228)$ when measured by the questionnaire and 12,457 (11,053-14,044) when measured by the accelerometer. Overall, PA levels measured by the questionnaire were lower than those measured by the accelerometer. With borderline significance, the total MET-min/wk measured by the questionnaire was higher in men than in women, but the total MET-min/wk measured by the accelerometer was higher in women than in men.

Table 2 shows the cross-classification of tertile groups of self-reported and accelerometer-assessed total MET-min/wk. The kappa coefficient was 0.16 in men and 0.19 in women, suggesting overall poor agreement. The strength of agreement between self-reported and accelerometer-assessed MET-min/wk is shown in Figure 1.

Table 3 shows correlations between the questionnaire and the

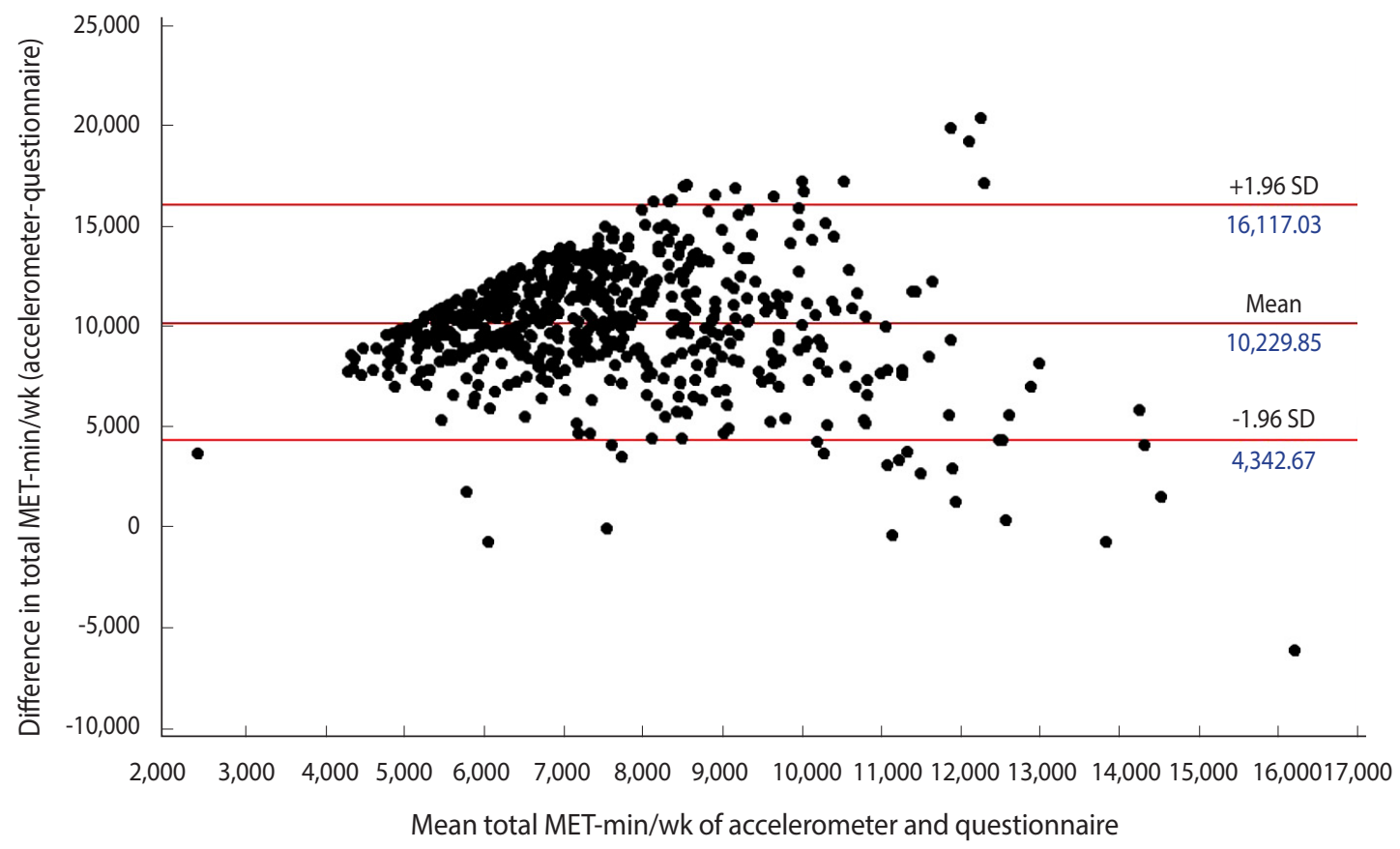

Figure 1. Bland-Altman plot for metabolic equivalent task minutes per week (MET-min/wk) assessed by a questionnaire and an accelerometer. Horizontal lines are drawn at the mean difference in total MET-min/wk, and at the limits of agreement. SD, standard deviation. 
Table 3. Correlations between self-reported and accelerometer-assessed PA per week

\begin{tabular}{|c|c|c|c|c|c|c|c|c|c|c|}
\hline \multirow{3}{*}{$\begin{array}{l}\text { Accelerometer-measured } \\
\text { PA (time) }\end{array}$} & \multicolumn{10}{|c|}{ Self-reported PA (time) } \\
\hline & \multicolumn{2}{|c|}{ Sitting } & \multicolumn{2}{|c|}{ Walking } & \multicolumn{2}{|c|}{ Moderate activity } & \multicolumn{2}{|c|}{ Vigorous activity } & \multicolumn{2}{|c|}{ Total MET-min/wk } \\
\hline & $r$ & $\mathrm{p}$-value & r & p-value & $r$ & $p$-value & $r$ & $p$-value & $r$ & $p$-value \\
\hline \multicolumn{11}{|l|}{ Total population $(n=623)$} \\
\hline Sedentary activity & 0.36 & $<0.001$ & -0.12 & 0.004 & -0.11 & 0.006 & 0.03 & 0.43 & -0.16 & $<0.001$ \\
\hline Light activity & -0.30 & $<0.001$ & 0.05 & 0.17 & 0.08 & 0.04 & -0.07 & 0.10 & 0.06 & 0.17 \\
\hline Moderate activity & -0.33 & $<0.001$ & 0.21 & $<0.001$ & 0.19 & $<0.001$ & 0.04 & 0.34 & 0.29 & $<0.001$ \\
\hline Vigorous activity & -0.08 & 0.04 & 0.09 & 0.02 & 0.11 & 0.007 & 0.20 & $<0.001$ & 0.22 & $<0.001$ \\
\hline Total MET-min/wk & -0.29 & $<0.001$ & 0.16 & $<0.001$ & 0.18 & $<0.001$ & 0.07 & 0.06 & 0.26 & $<0.001$ \\
\hline \multicolumn{11}{|l|}{ Men $(n=203)$} \\
\hline Sedentary activity & 0.36 & $<0.001$ & -0.21 & 0.002 & -0.19 & 0.008 & -0.15 & 0.04 & -0.35 & $<0.001$ \\
\hline Light activity & -0.23 & 0.001 & 0.07 & 0.30 & 0.10 & 0.17 & -0.02 & 0.79 & 0.12 & 0.10 \\
\hline Moderate activity & -0.28 & $<0.001$ & 0.21 & 0.003 & 0.24 & $<0.001$ & 0.16 & 0.02 & 0.37 & $<0.001$ \\
\hline Vigorous activity & -0.12 & 0.09 & 0.02 & 0.77 & 0.05 & 0.46 & 0.15 & 0.03 & 0.12 & 0.09 \\
\hline Total MET-min/wk & -0.23 & 0.001 & 0.12 & 0.08 & 0.21 & 0.003 & 0.10 & 0.15 & 0.27 & $<0.001$ \\
\hline \multicolumn{11}{|l|}{ Women $(n=420)$} \\
\hline Sedentary activity & 0.30 & $<0.001$ & -0.06 & 0.18 & -0.11 & 0.02 & 0.06 & 0.20 & -0.10 & 0.05 \\
\hline Light activity & -0.28 & $<0.001$ & 0.01 & 0.77 & 0.11 & 0.02 & $<0.01$ & 0.95 & 0.04 & 0.43 \\
\hline Moderate activity & -0.31 & $<0.001$ & 0.20 & $<0.001$ & 0.19 & $<0.001$ & 0.02 & 0.68 & 0.27 & $<0.001$ \\
\hline Vigorous activity & -0.12 & 0.02 & 0.14 & 0.003 & 0.12 & 0.01 & 0.17 & $<0.001$ & 0.26 & $<0.001$ \\
\hline Total MET-min/wk & -0.30 & $<0.001$ & 0.17 & $<0.001$ & 0.17 & $<0.001$ & 0.09 & 0.06 & 0.26 & $<0.001$ \\
\hline
\end{tabular}

$\mathrm{PA}$, physical activity; MET, metabolic equivalent task.

accelerometer when measuring the time participants spent engaged in different types of PA and MET-min/wk. In total population, the correlation coefficient between self-reported sitting time and accelerometer-assessed sedentary time was $0.36(\mathrm{p}<0.001)$, which was the highest correlation coefficient observed in the current study. The correlation coefficient between self-reported and accelerometer-assessed time was $0.20(\mathrm{p}<0.001)$ for vigorous activity and $0.19(\mathrm{p}<0.001)$ for moderate-intensity activity.

The correlation coefficient between self-reported and accelerometer-assessed MET-min/wk was $0.26(\mathrm{p}<0.001)$ in the total population (Table 4 ). The correlation did not significantly differ by gender, age, BMI, marital status, education, income, cognitive function, or depression. However, as age and depression scores increased, the correlation between self-reported and accelerometer-assessed PA tended to decrease ( $\mathrm{p}$ for trend in age and depression score $<0.001$, respectively). Additionally, when we investigated correlations between questionnaire- and accelerometer-assessed PA according to occupation (white collar, blue collar, and unemployed), no significant differences in the strength of the correlation among occupational groups were found (data not shown).

\section{DISCUSSION}

We examined the relationship between self-reported and accelerometer-assessed PA and whether the relationship differed by demographic and socioeconomic factors. Overall, less PA was measured by the questionnaire than by the accelerometer. This is probably because activities of short duration, for instance lasting less than 10 minutes, are unlikely to be captured by a questionnaire, but can be detected by an accelerometer. The mean time of vigorous activity in our study population was lower than in previous studies, but the total PA level (MET-min/wk) was similar [26]. It is possible that the participants responded incorrectly to questionnaire items about exercise intensity.

The overall correlation between self-reported and accelerometer-assessed PA in our study (total MET-min/wk, $r=0.26$ ) was relatively low, and the correlation decreased with increasing age and depression score. A significant difference was found in PA according to season, but there was no significant seasonal effect on the correlation between the 2 measurements (data not shown).

The correlation between questionnaire- and accelerometer-assessed PA in our study was similar to the results of previous studies. In a previous study with 1,270 Hong Kong Chinese participants, the overall Spearman correlation between IPAQ-assessed and accelerometer-assessed PA (MET-min/wk) ranged from 0.06 to 0.24 [27]. The Spearman correlation coefficient between questionnaire- and accelerometer-assessed PA was 0.33 (95\% confidence interval [CI], 0.30 to 0.36 ) in the Whitehall II study and 0.30 (95\% CI, 0.25 to 0.34 ) in the Rotterdam study [13,14]. The Whitehall II study also reported that the correlation between the 2 measurements was higher for more energetic activities [14]. However, our data showed that the highest correlation was found between questionnaire-assessed sitting time and accelerometerassessed sedentary activity time. The discrepancy between our re- 
Table 4. Spearman correlation between self-reported and accelerometer-assessed physical activity according to demographic and socioeconomic factors

\begin{tabular}{|c|c|c|c|c|c|}
\hline & \multirow{2}{*}{$\mathrm{n}(\%)$} & \multicolumn{3}{|c|}{ Total MET-min/wk } & \multirow{2}{*}{$p$ for trend } \\
\hline & & $r$ & p-value & $p$ for difference & \\
\hline Total population & $623(100)$ & 0.26 & $<0.001$ & & \\
\hline \multicolumn{6}{|l|}{ Gender } \\
\hline Men & $203(32.6)$ & 0.27 & $<0.001$ & 0.90 & $\mathrm{~N} / \mathrm{A}$ \\
\hline Women & $420(67.4)$ & 0.26 & $<0.001$ & & \\
\hline \multicolumn{6}{|l|}{ Age group (yr) } \\
\hline $30-39$ & $83(13.3)$ & 0.31 & 0.004 & 0.29 & $<0.001$ \\
\hline $40-49$ & $94(15.1)$ & 0.29 & 0.004 & & \\
\hline $50-59$ & $268(43.0)$ & 0.31 & $<0.001$ & & \\
\hline $60-64$ & $178(28.6)$ & 0.13 & 0.09 & & \\
\hline BMI $\left(\mathrm{kg} / \mathrm{m}^{2}\right)$ & & & & 0.10 & 0.37 \\
\hline$<22.9$ & $252(40.4)$ & 0.27 & $<0.001$ & & \\
\hline $23.0-24.9$ & $175(28.1)$ & 0.26 & $<0.001$ & & \\
\hline $25.0-29.9$ & $182(29.2)$ & 0.26 & $<0.001$ & & \\
\hline$\geq 30.0$ & $14(2.3)$ & 0.24 & 0.40 & & \\
\hline \multicolumn{6}{|l|}{ Marital status } \\
\hline Married/cohabiting & $544(87.3)$ & 0.23 & $<0.001$ & 0.80 & $\mathrm{~N} / \mathrm{A}$ \\
\hline Single & $79(12.7)$ & 0.41 & $<0.001$ & & \\
\hline \multicolumn{6}{|l|}{ Education } \\
\hline Secondary school or below & $318(51.0)$ & 0.24 & $<0.001$ & 0.81 & $\mathrm{~N} / \mathrm{A}$ \\
\hline University degree or more & $305(49.0)$ & 0.26 & $<0.001$ & & \\
\hline \multicolumn{6}{|l|}{ Income } \\
\hline Lower & $195(31.3)$ & 0.27 & $<0.001$ & 0.17 & 0.25 \\
\hline Middle & $193(31.0)$ & 0.34 & $<0.001$ & & \\
\hline Upper & $235(37.7)$ & 0.17 & 0.009 & & \\
\hline \multicolumn{6}{|l|}{ MMSE score } \\
\hline$<26$ & $225(36.1)$ & 0.33 & $<0.001$ & 0.34 & $\mathrm{~N} / \mathrm{A}$ \\
\hline$\geq 26$ & $398(63.9)$ & 0.22 & $<0.001$ & & \\
\hline \multicolumn{6}{|l|}{ BDI score } \\
\hline None (0-13) & $474(76.1)$ & 0.26 & $<0.001$ & 1.00 & $<0.001$ \\
\hline Mild (14-19) & $93(14.9)$ & 0.26 & 0.01 & & \\
\hline Moderate (20-28) & $43(6.9)$ & 0.21 & 0.17 & & \\
\hline Severe (29-63) & $13(2.1)$ & -0.23 & 0.45 & & \\
\hline
\end{tabular}

MET, metabolic equivalent task; BMI, body mass index; MSE, Mini-Mental State Estimation; BDI, Beck Depression Inventory-II; N/A, not applicable.

sults and those of that previous study may have been due to the use of a different type of questionnaires (the IPAQ vs. the Minnesota Leisure Time Physical Activity Questionnaire $[28,29]$ ) and differences in the characteristics of the study populations.

Regarding the influence of demographic and socioeconomic factors on the correlation between questionnaire- and accelerometer-assessed PA, most previous studies showed a higher correlation in men [12,16,30-32], younger people [27,30-32], and those with higher levels of education [14,27].

A study with Hong Kong Chinese participants reported that gender, age, job status (full-time worker or not), educational level, and obesity could influence the validity of the IPAQ, but did not appear to influence the correlation between IPAQ and accelerometer data [27]. In the Whitehall II study, the correlation was higher in people with a high educational level or occupational position than in people with a low educational level or occupational position [14]. In the Rotterdam study, people with high education had a greater correlation coefficient, and people with obesity, a higher disability score, and more depressive symptoms had a greater difference in the 2 measures [13].

In our data, older people and those with a higher depression score tended to have lower correlation coefficients than their younger or healthier counterparts. However, the correlation between questionnaire- and accelerometer-assessed PA did not differ by gender, marital status, household income, or MMSE score. The questionnaire survey showed that the oldest age group (aged over 60 years) had the highest level of PA (MET-min/wk), while the accelerometer test showed the lowest level of PA in the oldest age 
group (data not shown).

The possible reasons why the correlation between self-reported and questionnaire-assessed PA decreased as age increased include memory difficulties and cognitive problems, which are more prevalent in elderly adults. The questions of the IPAQ-Short Form that require the use of recognition memory are preferred over those that require recall $[33,34]$. However, in our study, cognitive function, as assessed by the MMSE score, was not associated with the correlation between self-reported and questionnaire-assessed PA. Additionally, there were few people who had cognitive problems in our study. Another possibility is that the open-ended response format of the IPAQ-Short Form can be difficult for elderly adults to complete accurately [35].

A previous study reported that the measurement method of PA is important when investigating associations between PA and depression [36]. Depressed persons show a response bias favoring the reporting of negative self-relevant information [37]. Reporting bias may therefore have influenced the current study.

Accelerometers have been often used in validation studies [12,32], but they are not a gold standard, since they measure the movement of only a single part of the body, but the resulting inferences are applied to the whole body. In addition, previous studies have proposed thresholds to define mild, moderate, and vigorous levels of PA and developed algorithms for detecting various types of PA $[21,38,39]$. However, there is no consensus on the best method, and considerable inconsistencies exist in results derived from different algorithms [14]. Measuring PA by questionnaire is the most cost-effective method, and questionnaires can be used to assess all types of PA and in large populations in epidemiological research [5]. Questionnaires can also assess PA for a relatively long time period. However, self-reported PA by questionnaire has several limitations, such as reporting and recall bias, as well as the inability to capture the absolute level of PA [12]. Activities of light intensity are hard to recall and might not be reported $[7,13]$. Furthermore, moderate or vigorous activities performed for a very short duration might not be recalled by the participants when they respond to the questionnaire [13]. Particular caution must be taken when using a questionnaire for young and elderly participants, as their memory can be incomplete [40,41]. In particular, older adults are more likely to engage in light- to moderate-intensity PA, which is the most difficult type of activity to assess through a questionnaire [42]. In addition, the IPAQ only includes activities of moderate or vigorous intensity carried out for more than an hour, which may explain the underestimation of PA in the questionnaire [13]. To redeem the limitations of the questionnaire, researchers have used motion sensors, such as pedometers or accelerometers, as an additional measurement for assessing PA in a free-living environment [43]. Accelerometers can record the acceleration associated with body movement, which can provide information on the duration and intensity of certain PAs [44]. Accelerometers include all PAs, including small bouts of activity (less than 5 minutes) and can avoid recall and response bias. Despite the advantages of using accelerometers, they are time-consuming and costly to apply in studies with a large-scale epidemiological research design. Additionally, PA measured with a wrist-worn accelerometer can be underestimated when an individual engages in PA with the wrist fixed, such as carrying a briefcase, or PA that only involves the legs, such as cycling [45]. Additionally, when collecting PA data using an accelerometer, caution should be taken regarding variation in participants' compliance in terms of wearing the device and seasonal variation reflecting the possibility of water-based activities [5]. Because both accelerometers and questionnaires have advantages and disadvantages, using both measures is recommended as a way to collectively measure an individual's PA. Further studies are also required to develop a better understanding of the association between questionnaire- and accelerometer-assessed PA.

Our study contains several strengths. First, we used a validated accelerometer and questionnaire; thus, our results can be compared with previous studies that used the same assessment tools. Second, our study showed relatively high compliance for accelerometer wearing. Third, our study population consisted of a large number of community-dwelling adults from a large populationbased cohort.

However, our study also had some limitations. First, the PA data derived from the accelerometer and questionnaire were not obtained in the same week. The accelerometer measurements were made over a 7-day period after participants completed the questionnaire. This might have contributed to the low correlation coefficients between self-reported and accelerometer-assessed PA in the current study. Second, as the data were drawn from a subsample of the CMERC cohort, which consists of communitydwelling healthy people aged 30-65 years without a history of CVD, who might have had a different PA pattern from those who are less active. Furthermore, those who were extremely active might have felt too much pressure from the accelerometer and refused to participate because the accelerometer could interfere with their activity and there was a risk that the device would break during PA [27]. However, in our sensitivity analysis, there were no significant differences in characteristics such as gender, marital status, education level, depression score, BMI, and blood pressure between people who participated in the accelerometer component of the study and those who refused to participate (data not shown). However, the mean age and MMSE scores of those who chose to participate in the accelerometer component were higher than those of individuals who did not participate. A third limitation is the lack of randomization. However, our study utilized a community-based cohort design, which represents realworld circumstances well [46]. Fourth, we used a wrist-worn accelerometer due to expected higher compliance [47]. Previous studies have typically used hip-worn accelerometers in order to better reflect lower body movements $[47,48]$. The National Health and Nutrition Examination Survey, which conducts surveillance of PA in the US population, previously used a uniaxial accelerometer worn on the hip (2003-2004 and 2005-2006), but changed its protocol and asked participants to wear a triaxial accelerometer 
on the wrist during recent surveys (2011-2014) among persons aged over 6 years $[47,49]$. Also, several studies reported that hip and wrist-worn accelerometers were moderately correlated in adults and adolescents $[49,50]$. Finally, although our results are in accordance with those of previous studies that used different instruments and a different type of accelerometer, our results might not be generalizable to other instruments.

In conclusion, we found a low correlation between self-reported and accelerometer-assessed PA among healthy Korean adults, and the correlation decreased with age and depression score. Future studies assessing PA using questionnaires and/or accelerometers should take these results into account.

\section{ACKNOWLEDGEMENTS}

This work was supported by a grant of the Korea Health Technology R\&D Project through the Korea Health Industry Development Institute (KHIDI), funded by the Ministry of Health and Welfare, Republic of Korea (grant no. HI13C0715). This research was also supported by the Bio \& Medical Technology Development Program of the National Research Foundation, funded by the Ministry of Science and ICT (grant no. NRF-2017M3A9E8029714).

\section{CONFLICT OF INTEREST}

The authors have no conflicts of interest to declare for this study.

\section{ORCID}

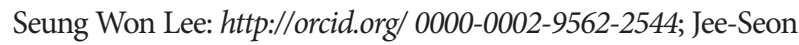
Shim: http://orcid.org/0000-0002-8671-3153; Bo Mi Song: http:// orcid.org/0000-0003-3181-5803; Ho Jae Lee: http://orcid.org/00000001-6739-2780; Hye Yoon Bae: https://orcid.org/0000-0002-60581930; Ji Hye Park: http://orcid.org/0000-0002-1004-2496; Hye Rin Choi: $h t t p: / / o r c i d . o r g / 0000-0002-4373-2581$; Jae Won Yang: http:// orcid.org/0000-0001-8119-2574; Ji Eun Heo: http://orcid.org/00000001-6449-5856; So Mi Jemma Cho: http://orcid.org/0000-00032460-3335; Ga Bin Lee: https://orcid.org/0000-0001-6574-1414; Diana Huaman Hidalgo: https://orcid.org/0000-0003-2874-5875; Tae-Hoon Kim: https://orcid.org/0000-0003-4200-3456; Kyung Soo Chung: http://orcid.org/0000-0003-1604-8730; Hyeon Chang Kim: http://orcid.org/0000-0001-7867-1240

\section{REFERENCES}

1. Nocon M, Hiemann T, Müller-Riemenschneider F, Thalau F, Roll S, Willich SN. Association of physical activity with all-cause and cardiovascular mortality: a systematic review and meta-analysis. Eur J Cardiovasc Prev Rehabil 2008;15:239-246.

2. Hu FB, Leitzmann MF, Stampfer MJ, Colditz GA, Willett WC, Rimm EB. Physical activity and television watching in relation to risk for type 2 diabetes mellitus in men. Arch Intern Med 2001; 161:1542-1548.
3. Gregg EW, Pereira MA, Caspersen CJ. Physical activity, falls, and fractures among older adults: a review of the epidemiologic evidence. J Am Geriatr Soc 2000;48:883-893

4. Sangrajrang S, Chaiwerawattana A, Ploysawang P, Nooklang K, Jamsri P, Somharnwong S. Obesity, diet and physical inactivity and risk of breast cancer in Thai women. Asian Pac J Cancer Prev 2013;14:7023-7027.

5. Skender S, Ose J, Chang-Claude J, Paskow M, Brühmann B, Siegel EM, et al. Accelerometry and physical activity questionnaires - a systematic review. BMC Public Health 2016;16:515.

6. Sallis JF, Saelens BE. Assessment of physical activity by self-report: status, limitations, and future directions. Res Q Exerc Sport 2000; 71 Suppl 2:1-14.

7. Schmid D, Ricci C, Leitzmann MF. Associations of objectively assessed physical activity and sedentary time with all-cause mortality in US adults: the NHANES study. PLoS One 2015;10:e0119591.

8. Ensrud KE, Blackwell TL, Cauley JA, Dam TT, Cawthon PM, Schousboe JT, et al. Objective measures of activity level and mortality in older men. J Am Geriatr Soc 2014;62:2079-2087.

9. Frost C, Thompson SG. Correcting for regression dilution bias: comparison of methods for a single predictor variable. J R Stat Soc Ser A Stat Soc 2000;163:173-189.

10. Washburn RA. Assessment of physical activity in older adults. Res Q Exerc Sport 2000;71 Suppl 2:79-87.

11. Scheers T, Philippaerts R, Lefevre J. Assessment of physical activity and inactivity in multiple domains of daily life: a comparison between a computerized questionnaire and the SenseWear Armband complemented with an electronic diary. Int J Behav Nutr Phys Act 2012;9:71.

12. Prince SA, Adamo KB, Hamel ME, Hardt J, Connor Gorber S, Tremblay M. A comparison of direct versus self-report measures for assessing physical activity in adults: a systematic review. Int J Behav Nutr Phys Act 2008;5:56.

13. Koolhaas CM, van Rooij FJ, Cepeda M, Tiemeier H, Franco OH, Schoufour JD. Physical activity derived from questionnaires and wrist-worn accelerometers: comparability and the role of demographic, lifestyle, and health factors among a population-based sample of older adults. Clin Epidemiol 2017;10:1-16.

14. Sabia S, van Hees VT, Shipley MJ, Trenell MI, Hagger-Johnson G, Elbaz A, et al. Association between questionnaire- and accelerometer-assessed physical activity: the role of sociodemographic factors. Am J Epidemiol 2014;179:781-790.

15. Shim JS, Song BM, Lee JH, Lee SW, Park JH, Choi DP, et al. Cardiovascular and Metabolic Diseases Etiology Research Center (CMERC) cohort: study protocol and results of the first 3 years of enrollment. Epidemiol Health 2017;39:e2017016.

16. Chun MY. Validity and reliability of Korean version of international physical activity questionnaire short form in the elderly. Korean J Fam Med 2012;33:144-151.

17. Craig CL, Marshall AL, Sjöström M, Bauman AE, Booth ML, Ainsworth $\mathrm{BE}$, et al. International physical activity questionnaire: 12country reliability and validity. Med Sci Sports Exerc 2003;35:13811395. 
18. Ainsworth BE, Haskell WL, Whitt MC, Irwin ML, Swartz AM, Strath SJ, et al. Compendium of physical activities: an update of activity codes and MET intensities. Med Sci Sports Exerc 2000; 32(9 Suppl):S498-S504.

19. Oh JY, Yang YJ, Kim BS, Kang JH. Validity and reliability of Korean version of International Physical Activity Questionnaire (IPAQ) Short Form. J Korean Acad Fam Med 2007;28:532-541 (Korean).

20. GENEActiv. How to use macros; 2014 [cited 2017 May 4]. Available from: https://open.geneactiv.org/geneactiv_macros.html.

21. Esliger DW, Rowlands AV, Hurst TL, Catt M, Murray P, Eston RG. Validation of the GENEA accelerometer. Med Sci Sports Exerc 2011;43:1085-1093.

22. Kim TH, Jhoo JH, Park JH, Kim JL, Ryu SH, Moon SW, et al. Korean version of mini mental status examination for dementia screening and its' short form. Psychiatry Investig 2010;7:102-108.

23. Kukull WA, Larson EB, Teri L, Bowen J, McCormick W, Pfanschmidt ML. The Mini-Mental State Examination score and the clinical diagnosis of dementia. J Clin Epidemiol 1994;47:10611067.

24. Lim SY, Lee EJ, Jeong SW, Kim HC, Jeong CH, Jeon TY, et al. The validation study of Beck Depression Scale 2 in Korean version. Anxiety Mood 2011;7:48-53 (Korean).

25. Smarr KL, Keefer AL. Measures of depression and depressive symptoms: Beck Depression Inventory-II (BDI-II), Center for Epidemiologic Studies Depression Scale (CES-D), Geriatric Depression Scale (GDS), Hospital Anxiety and Depression Scale (HADS), and Patient Health Questionnaire-9 (PHQ-9). Arthritis Care Res (Hoboken) 2011;63 Suppl 11:S454-S466.

26. Dyrstad SM, Hansen BH, Holme IM, Anderssen SA. Comparison of self-reported versus accelerometer-measured physical activity. Med Sci Sports Exerc 2014;46:99-106.

27. Lee PH, Yu YY, McDowell I, Leung GM, Lam TH, Stewart SM. Performance of the international physical activity questionnaire (short form) in subgroups of the Hong Kong chinese population. Int J Behav Nutr Phys Act 2011;8:81.

28. Jacobs DR Jr, Ainsworth BE, Hartman TJ, Leon AS. A simultaneous evaluation of 10 commonly used physical activity questionnaires. Med Sci Sports Exerc 1993;25:81-91.

29. Richardson MT, Leon AS, Jacobs DR Jr, Ainsworth BE, Serfass R. Comprehensive evaluation of the Minnesota leisure time physical activity questionnaire. J Clin Epidemiol 1994;47:271-281.

30. Ferrari P, Friedenreich C, Matthews CE. The role of measurement error in estimating levels of physical activity. Am J Epidemiol 2007; 166:832-840.

31. Friedenreich CM, Courneya KS, Neilson HK, Matthews CE, Willis G, Irwin M, et al. Reliability and validity of the past year total physical activity questionnaire. Am J Epidemiol 2006;163: 959-970.

32. Cust AE, Smith BJ, Chau J, van der Ploeg HP, Friedenreich CM, Armstrong BK, et al. Validity and repeatability of the EPIC physical activity questionnaire: a validation study using accelerometers as an objective measure. Int J Behav Nutr Phys Act 2008;5:33.
33. Stewart AL, Mills KM, King AC, Haskell WL, Gillis D, Ritter PL. CHAMPS physical activity questionnaire for older adults: outcomes for interventions. Med Sci Sports Exerc 2001;33:11261141.

34. Stewart A, Hays RD. Conceptual measurement, and analytical issues in assessing health status in older populations. In: Hickey T, Speers MA, Prohaska TR, editors. Public health and aging. Baltimore: Johns Hopkins University; 1997, p. 163-189.

35. Washburn RA, Smith KW, Jette AM, Janney CA. The Physical Activity Scale for the Elderly (PASE): development and evaluation. J Clin Epidemiol 1993;46:153-162.

36. McKercher CM, Schmidt MD, Sanderson KA, Patton GC, Dwyer T, Venn AJ. Physical activity and depression in young adults. Am J Prev Med 2009;36:161-164.

37. Zuroff DC, Colussy SA, Wielgus MS. Selective memory and depression: a cautionary note concerning response bias. Cogn Ther Res 1983; 7:223-231.

38. Troiano RP, Berrigan D, Dodd KW, Mâsse LC, Tilert T, McDowell M. Physical activity in the United States measured by accelerometer. Med Sci Sports Exerc 2008;40:181-188.

39. Zhang S, Murray P, Zillmer R, Eston RG, Catt M, Rowlands AV. Activity classification using the GENEA: optimum sampling frequency and number of axes. Med Sci Sports Exerc 2012;44:22282234.

40. Baranowski T, Dworkin RJ, Cieslik CJ, Hooks P, Clearman DR, Ray L, et al. Reliability and validity of self report of aerobic activity: Family Health Project. Res Q Exerc Sport1984;55:309-317.

41. Sallis JF. Self-report measures of children's physical activity. J Sch Health 1991;61:215-219.

42. Washburn RA, Jette AM, Janney CA. Using age-neutral physical activity questionnaires in research with the elderly. J Aging Health 1990;2:341-356.

43. Troiano RP. A timely meeting: objective measurement of physical activity. Med Sci Sports Exerc 2005;37(11 Suppl):S487-S489.

44. Westerterp KR. Physical activity assessment with accelerometers. Int J Obes Relat Metab Disord 1999;23 Suppl 3:S45-S49.

45. Rosenberger ME, Haskell WL, Albinali F, Mota S, Nawyn J, Intille S. Estimating activity and sedentary behavior from an accelerometer on the hip or wrist. Med Sci Sports Exerc 2013;45:964975.

46. Manser CN, Bachmann LM, Brunner J, Hunold F, Bauerfeind P, Marbet UA. Colonoscopy screening markedly reduces the occurrence of colon carcinomas and carcinoma-related death: a closed cohort study. Gastrointest Endosc 2012;76:110-117.

47. Troiano RP, McClain JJ, Brychta RJ, Chen KY. Evolution of accelerometer methods for physical activity research. Br J Sports Med 2014;48:1019-1023.

48. Jefferis BJ, Sartini C, Shiroma E, Whincup PH, Wannamethee SG, Lee IM. Duration and breaks in sedentary behaviour: accelerometer data from 1566 community-dwelling older men (British Regional Heart Study). Br J Sports Med 2015;49:1591-1594.

49. Kamada M, Shiroma EJ, Harris TB, Lee IM. Comparison of physical activity assessed using hip- and wrist-worn accelerometers. 
Gait Posture 2016;44:23-28.

50. Scott JJ, Rowlands AV, Cliff DP, Morgan PJ, Plotnikoff RC, Lubans DR. Comparability and feasibility of wrist- and hip-worn acceler- ometers in free-living adolescents. J Sci Med Sport 2017;20:11011106. 